\title{
Ontology-based Facilitation Support Tool for Group Decision Making
}

\author{
Abdelkader Adla, Bakhta Nachet \\ Department of Computer Science \\ University of Oran 1 Ahmed Ben Bella \\ Oran, Algeria \\ \{adla.abdelkader, nachet bakhta\}@univ-oran.dz
}

\begin{abstract}
The need for Group Decision Making (GDM) techniques and support is greater than ever before. In the group decision making process, the alternatives amongst which a decision must be made can range from a few to a few thousand; the facilitator (or the decision makers) need(s) to narrow the possibilities down to a reasonable number, and categorize and classify alternatives. Even when this is not the case, facilitation support, such as ontology-based frameworks potentially offer these capabilities and can assist the decision-maker in presenting the alternatives in a form that facilitates the decision. In this research an ontology base approach is developed to facilitate organizing alternatives. The resulting alternatives organizing tool is based on two ontologies. These two ontologies are supplementary and each one ensures an aspect of the decision organizing. They have been built using the Web Ontology Language (OWL) which facilitates the sharing and integration of decision-making information between multiple decision makers.
\end{abstract}

Keywords - GDSS, ontology of the domain of application, ontology of domain, organization of alternatives, OWL

\section{INTRODUCTION}

The need for Group Decision Making (GDM) techniques and support is greater than ever before. This is due to the complexity of business relationships, the greater number of decision makers and organizations that are involved in the decision process, online access to multiple external information sources, and the decreasing in the time allowed for decision making.

In the group decision making process, the alternatives amongst which a decision must be made can range from a few to a few thousand [1][2]. The facilitator (or the decision makers) need(s) to narrow the possibilities down to a reasonable number, and categorize and classify alternatives, especially where the alternatives can be put into numerical terms. Even when this is not the case, facilitation support, such as ontology-based frameworks potentially offer these capabilities and can assist the decision-maker in presenting the alternatives in a form that facilitates the decision.

In this research, an ontology based approach is developed to facilitate organizing alternatives during the group decision making process. The alternatives organizing tool is based on two ontologies: application-domain ontology and domain ontology.

The first ontology will allow structuring all documented possible decisions by specifying semantic inter-relations. The domain ontology defines the objects of the domain as well as their inter-relations. This second ontology will ensure another aspect of the generalization link between decisions. As a result, these two ontologies are supplementary and each one ensures an aspect of the decision organizing.

We have built the ontologies using the Web Ontology Language (OWL) which facilitates the sharing and integration of decision-making information between multiple decision makers via the Web and Description Logic.

The remainder of this article is structured as follows: a background on group decision making and decision support is given in section 2 . The section 3 presents related works. In the section 4, we develop our ontology-based approach to organize alternatives decision in the group decision making process. Section 5 is devoted to a detailed presentation of the developed ontologies to facilitate the alternatives organizing, followed par an illustration with an example in section 6. Finally, in section 7 we conclude and give future work.

\section{BACKGROUND}

Decision aid and decision making have greatly changed with the emergence of information and communication technology (ICT). Decision makers are now far less statically located; on the contrary they play the role in a distributed way. This fundamental methodological change creates a new set of requirements: distributed group decision making is necessarily based on incomplete data, it must be possible at any moment, and it might be necessary to interrupt a decision process and to provide another, more viable decision. "Distributed group decision making" means that several entities (humans and machines) cooperate to 
reach an acceptable decision, and that these entities are distributed and possibly mobile along networks [1].

In [1], the authors consider the paradigm of distributed group decision-support systems, in which several decisionmakers who deal with partial, uncertain, and possibly exclusive information must reach a common decision. To this end, the use of a group system makes possible the collaboration of distant decision makers. The cooperative work so initiated can be synchronous or asynchronous. A small group or a whole organization can be supported. The application can be carried in several sites over a common information base. The networked decision makers work together to solve a particular problem although they might neither be present at the same time in the same place nor constitute a permanent organization. Thus, decision-makers can evaluate and rank alternatives, determine the implications of offers, maintain negotiation records, and concentrate on issues instead of personalities.

Experience with group decision making has shown that an on-line "meeting" is generally used to represent a group decision process for the specific problem at hand and a recurring pattern of three stages occurs in the group decision process [1]. These three process phases are: Pre-meeting, during meeting, and post- meeting (Fig. 1).

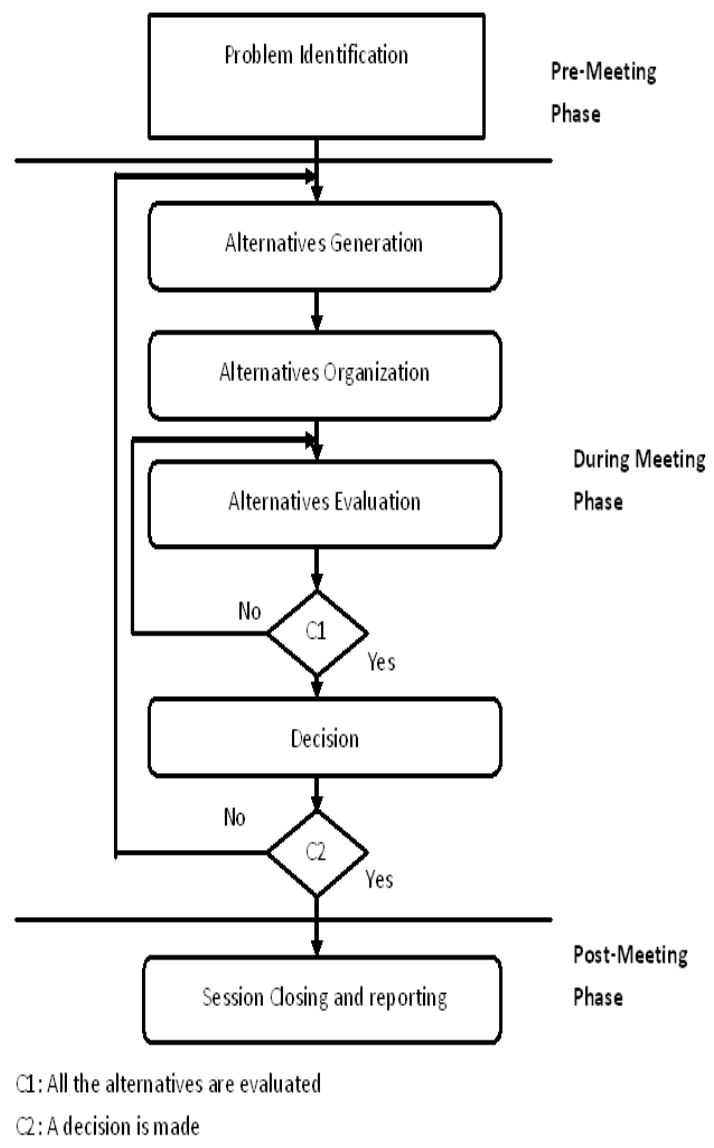

Figure 1 : Group Decision Making Process Model [ADLA 10]
The group decision model assumes that decision-makers are located in different places. A computer network is presumed that connects these different locations of participants. The decision-making process is controlled by a facilitator. The facilitator initiates, prepares the phases of the decision making process. He defines the issue(s) for decision and organizes the human group of decision makers for the decision-making process. His responsibility is to distribute the results among the participants after the decision-making. During the process, the mediator has a principal responsibility for the convergence of decision making process. He is responsible for the complete process and its deliverable, namely the decision.

In "during meeting" phase, a group can generate many alternatives in a short period of time. These alternatives may be similar or duplicated that need to be merged. The redundant alternatives can be retrieved for the facilitator to review, and then they can be merged or deleted. Idea organization in a distributed environment is mainly the facilitator's responsibility. It can be a very challenging task for the facilitator.

\section{RELATED WORK}

Ontologies are used as part of the improvement of the management of an organizational memory. In this perspective, ontology is mainly used to manage large case bases by facilitating their storage, representation and information semantic retrieval. Among the systems which use this aspect of ontologies in DSS, we cite the platform PROTEUS [3]. In the same context, the tool TextViz [4] is used information semantic retrieval in the field of car diagnosis. The ontology in that case, represents the knowledge concerning the breakdowns diagnosis. In [5], the authors propose an ontology of gas turbine and a reasoning tool based on that ontology

In [6], the author develops an ontology- based system to support the risks analysis in industrial domain. This system used resources indexing and a case base reasoning. Also in [7], the authors developed domain ontology to assist jurists during the juridical problem solving.

Overall the research in [8] provides the first attempt at documenting, storing, and retrieving engineering design decisions using ontologies and provides the foundation for the development of a more comprehensive decision support framework.

\section{THE ONTOLOGY BASED APPROACH FOR ALTERNATIVES ORGANIZING IN GROUP DECISION MAKING}

The alternatives proposed by the decision-makers can contain decisions which are:

- Redundant: the alternatives are syntactically identical;

- Synonyms: the alternatives are syntactically different, but semantically identical; 
- Conflicting: two contradictory or conflicting alternatives mean that the application of one is incompatible with the application of the other;

- Generic: an alternative may be more general than another. In this case, the application of the most general includes the application of the most specific;

These alternatives must be organized before being evaluated and thus enabling the decision choice. Our work consists to organize these alternatives. The alternative organizing contributes to retrieve and remove all the redundant, conflicting and synonymous decisions. Besides, when an alternative is more general than another, both the alternatives are presented to the decision-makers and it is their duty to choose one.

The main role of the organizing tool is to allow identifying semantic relationships between decisions then to present them to the decision-makers who will have the duty to decide among the suggested alternatives which will be removed and which have to be kept based on their expertise and the semantic relationships existing between the generated decisions.

\section{THE Proposed ONTOLOGIES}

The purpose of our work is to integrate an organizing tool (Fig. 2) into a Group Decision Support System (GDSS) to support the facilitator during the alternatives organizing stage in the group decision making process. Our ontology based approach to support alternatives organizing uses two ontologies:

1) Application domain ontology: It will be used in the alternatives organizing by the group of the decision-makers. It is a conceptual ontology where each object represents an alternative decision proposed by a decision-maker as a solution to the breakdown diagnosis problem. The application domain ontology specifies all decisions and the relations between them. Indeed, two decisions which can seem at first glance semantically close can be contradictory or incompatible in the context of the diagnosis of breakdowns application. This is why it is necessary to consider relations between decisions according to their effects on a particular task, i.e. the equipment maintenance, and not analyze a decision upon its syntactic expression based on the domain ontology.

2) Domain ontology: This ontology specifies concepts which are the equipment components. Relations between these concepts are of aggregation and inclusion. In effect a component which is included (directly or indirectly) in another is linked to the latter by a semantic relation of generalization. Domain ontology of the equipment concerns the vocabulary used in the expression of the decisions in terms of equipment components. The domain ontology is considered to be an explicit specification of concepts relating to the equipment maintenance as well as the relations existing between these concepts.
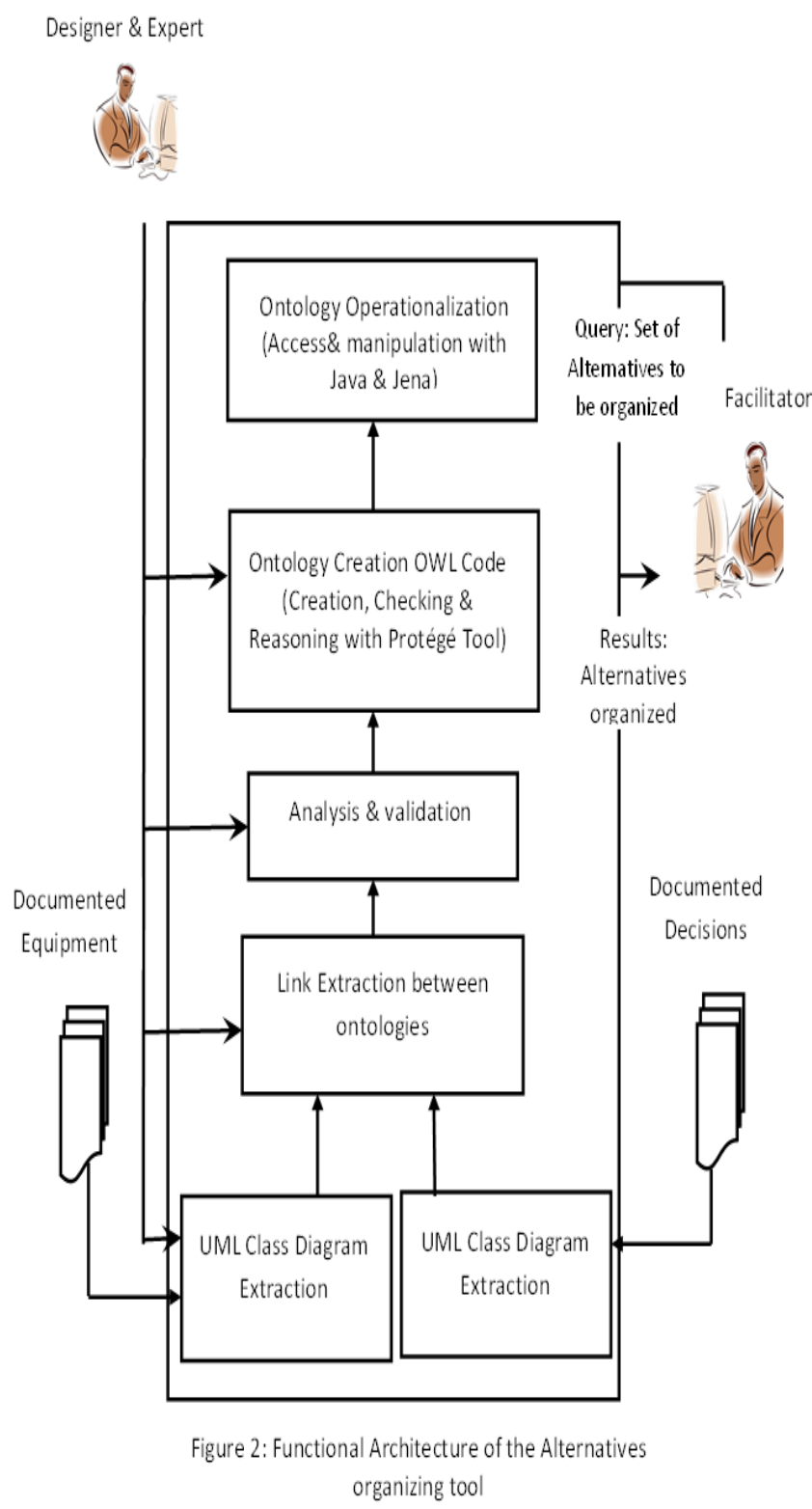

The link between both ontologies is materialized by the fact that in the application domain ontology each decision is indexed by one or several objects (components) implied by this decision. The use of two distinct but smoothly coupled ontologies will enable to infer new useful knowledge for the alternatives organizing task. Both ontologies must be fully developed. The general approach cited in [9] is adopted to develop both ontologies (Fig. 3). The three stages of the approach (conceptualization, ontologization and operationalization) are in general preceded of requirement analysis and knowledge domain delimitation. This process must however be entirely validated by a human expert. 


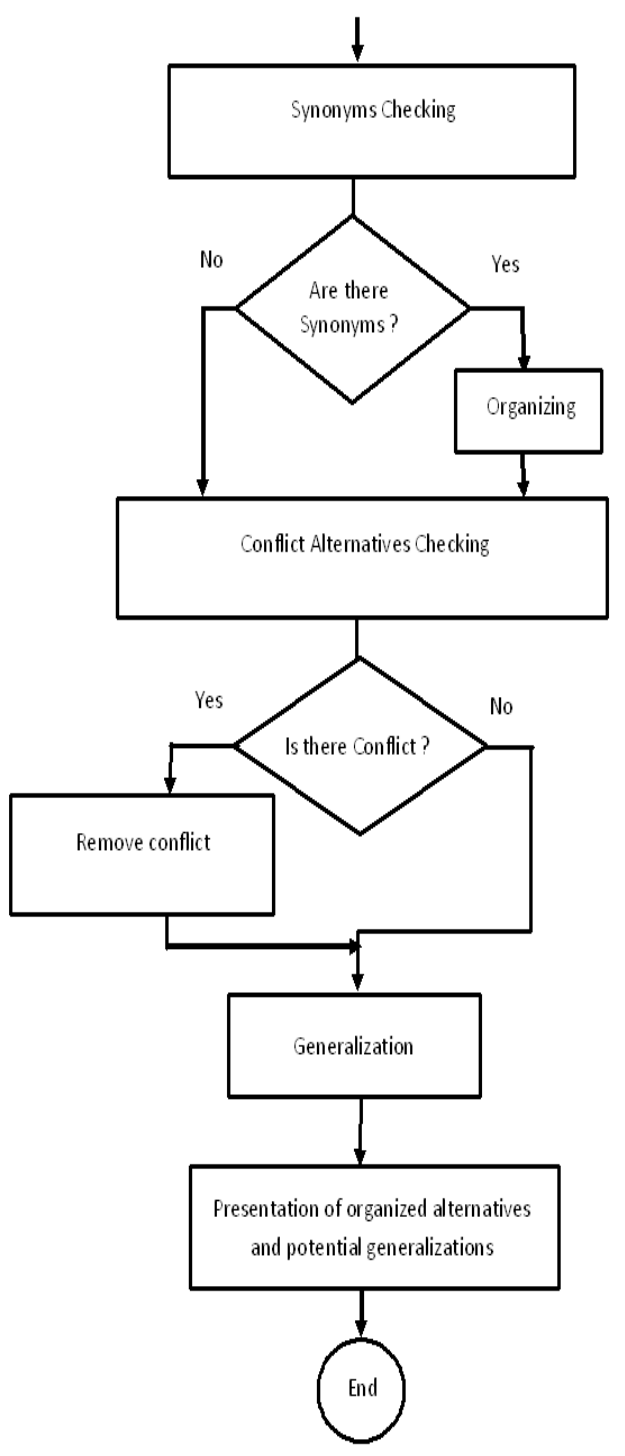

Figure 3: General functioning diagram of the alternatives organizing tool

We consider the breakdowns diagnosis application in a complex industrial system. In this kind of systems, decisions are known and listed in an appropriate documentation. The decision-makers who are experts in their domains propose decisions as possible solutions to the problem. Faced with the huge amount of alternatives decisions suggested by the decision makers, the facilitator has to come to a consensual decision. The integration of an organizing tool in the GDSS is the first stage in preparing the decision choice. In this regard, the tool is useful and will give a significant support to the facilitator.

\section{A. Conceptualization}

This stage consists in representing ontology by a conceptual model in a high level of abstraction. The used conceptual model represents the concept classes and their instances. We use UML class diagram to represent the conceptual models of ontology (Fig. 4) (Fig. 5). The ontology models allow representing domain concepts of classes and relations between the classes. Every concept or instance may be identified by URI. These models will be of use as inputs of the ontoligization stage.

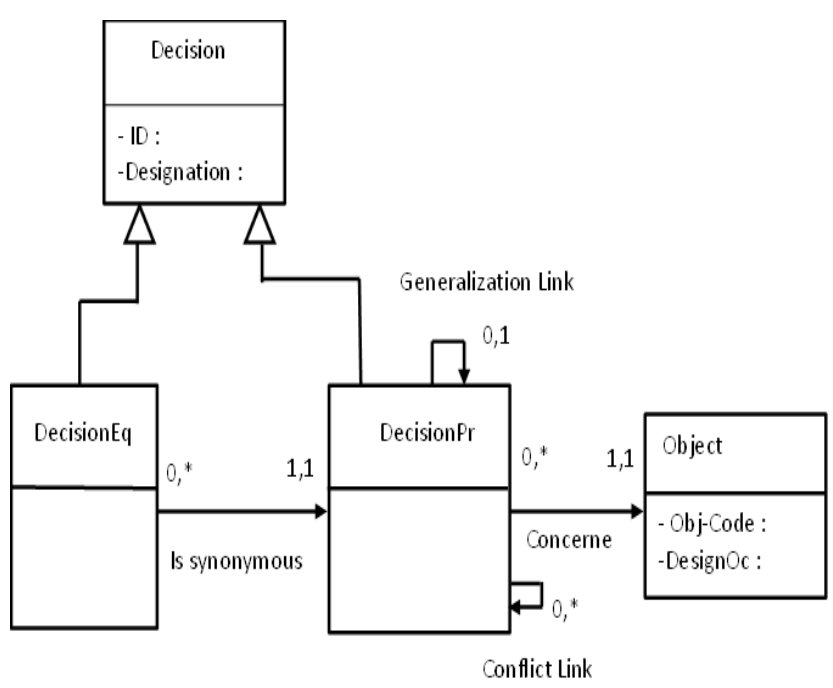

Figure 4: Conceptual Model of the Application domain Ontology

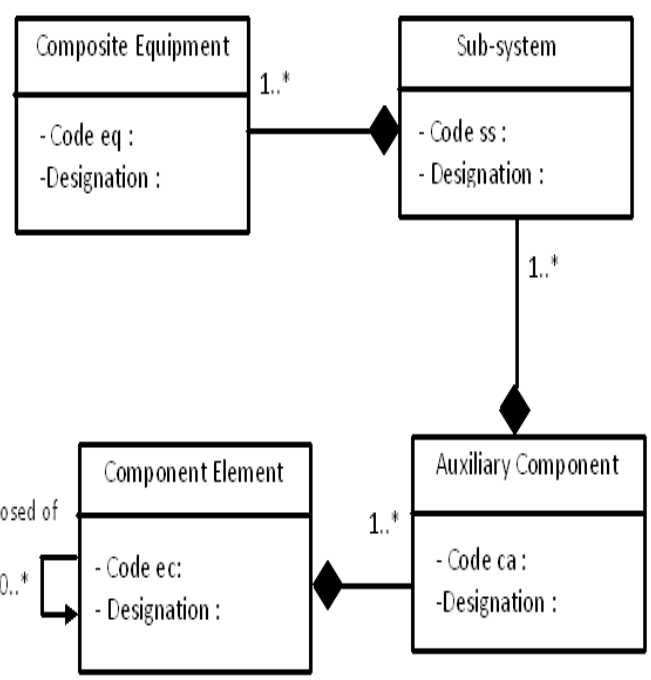

Figure 5: Conceptual Model of the domain ontology 
1) Application domain ontology: We define four classes:

a) Decision class: represents decisions;

b) DecisionEq class: represents equivalent decisions; it is a sub-class of the decision class. Several equivalent decisions can be related by a synonymy link with a main decision. These equivalent decisions are all different expressing forms of the same decision. This group of decisions is represented by one decision in the main decisions class which contains no semantic redundancy;

c) DecisionPr class: represents all main decisions which are interrelated semantically; it is a sub-class of the decision class;

d) Objetconcerned: represents objects (components) concerned by decisions.

We define three types of relations:

a) Conflict relations: links a main decision with all the main decisions which are conflicting. A main decision can have several contradiction links. This relation is symmetrical

b) Generalization relation: links a decision with its generic decisions. This relation is used to identify a first form of generalization between decisions inferred from the application domain ontology. Example: in the equipment maintenance, replacing a component is more general than repairing it. This relation is transitive;

c) Synonymy relation: a group of equivalent decisions is represented by one main decision. So, this relation will be used to identify synonymies between decisions. This relation is functional.

2) Domain ontology: We define four classes:

a) Composite equipment class: represents a composite equipment to maintain

b) Sub-system class: represents the sub-systems which compose an equipment;

c) Auxiliary component class: represents all auxiliary components which compose the sub-systems;

d) Component element class: represents the elements which compose the auxiliary components.

As for the semantic relations, we define four ones:

a) "Is composed of" relation: links the instances of Component element class. This link expresses the relation of composition of a component in another. This relation is transitive; it used to identify a second form of generalization between decisions inferred from the domain ontology of the equipment to maintain and uses the relation « is composed of ».

b) Aggregation type relations: The composite equipment is formed of a group of sub-systems; each of which is formed in return of a group of auxiliary components. Each of the latter contains a group of elements components.

\section{B. Ontologization}

Ontologization consists of formalizing conceptual models developed at the previous stage, as far as possible. We use OWL (Ontology Web Language) [10] as formalizing language of our ontologies. OWL is a developing information technology of the Semantic Web and is based in Description Logic (DL) [11]. Description logic is a family of knowledge representation languages used to formally represent knowledge of a domain in a structured manner. OWL represents ontology by building hierarchies of classes which describe the concepts in a domain and the properties which relate these classes to each other.

The creation of our ontologies is done using Protégé Ontology Editor which is an ontology development tool developed by Stanford Medical Informatics. [12]. This allowed the classes and properties to be easily created in an OWL-DL representation. We have also used it to check on our ontologies and the inconsistencies thanks to the reasoner FACT++. It allows as well inferring new knowledge from semantic relations. Then, we generated our ontologies in OWL format.

Example of the individual decision "change_the_parvexvariator" :

<owl:NamedIndividual

rdf:about="http://www.ontoproject.org/ontologydecision\#ch ange_the_parvex-variator">

<rdf:type rdf:resource"http://www.ontoproject.org/ ontologydecision \#Prdecision"/>

$<$ ID rdf:datatype="\&xsd;int" >19</ID>

$<$ Designation rdf:datatype $=" \& x s d$;string" $>$ the variator

is faulty, it must be replaced</Designation $>$

$<$ general

rdf:resource="http://www.ontoproject.org/ontologydecision \#check_the_connection_of_the_variator_plug"/>

<concern rdf:resource="http:// www.ontoproject.org/ ontologydecision \#parvex_variator"/>

$</$ owl:NamedIndividual>

Fig.6 depicts partial view of the application domain ontology. The URI base is:"http://www.ontoproject.org/ ontologydecision".

Example of the individual Component element "parvexvariator"

<owl:NamedIndividual

rdf:about="http://www.ontoproject.org/ontologyequipment\# parvex-variator">

<rdf:type

rdf:resource="http://www.ontoproject.org/ontologyequipme nt\#Componentelement"/>

$<$ Codeec rdf:datatype $=" \& x s d ;$ int $">7</$ Codeec $>$

$<$ Designation rdf:datatype="\&xsd;string" $>$ searching a label for parvex variator $</$ Designation $>$ 
<iscomposed

rdf:resource="http://www.ontoproject.org/ontologyequ ipment\#CAN-netwok"/>

$<$ iscomposed

rdf:resource="http://www.ontoproject.org/ontologyequ ipment\#axial-variator"/>

$<$ iscomposed

rdf:resource="http://www.ontoproject.org/ontologyequ ipment\#brushless-motor"/>

$<$ iscomposed

rdf:resource="http://www.ontoproject.org/ontologyequ ipment\#communication-network"/>

$<$ iscomposed

rdf:resource="http://www.ontoproject.org/ontologyequ ipment\#internal_fuse"/>

$</$ owl:NamedIndividual $>$

Fig. 7 depicts partial view of the equipment domain ontology. The URI base is:

http://www.ontoproject.org/ontologycomponents".

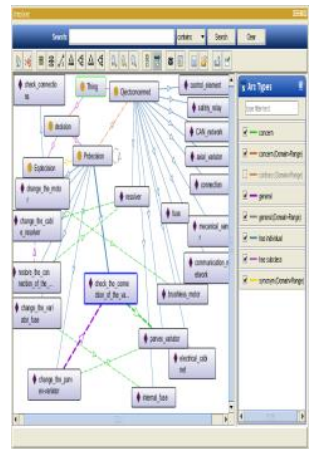

Fig. 6. Partial view of the application domain ontology.

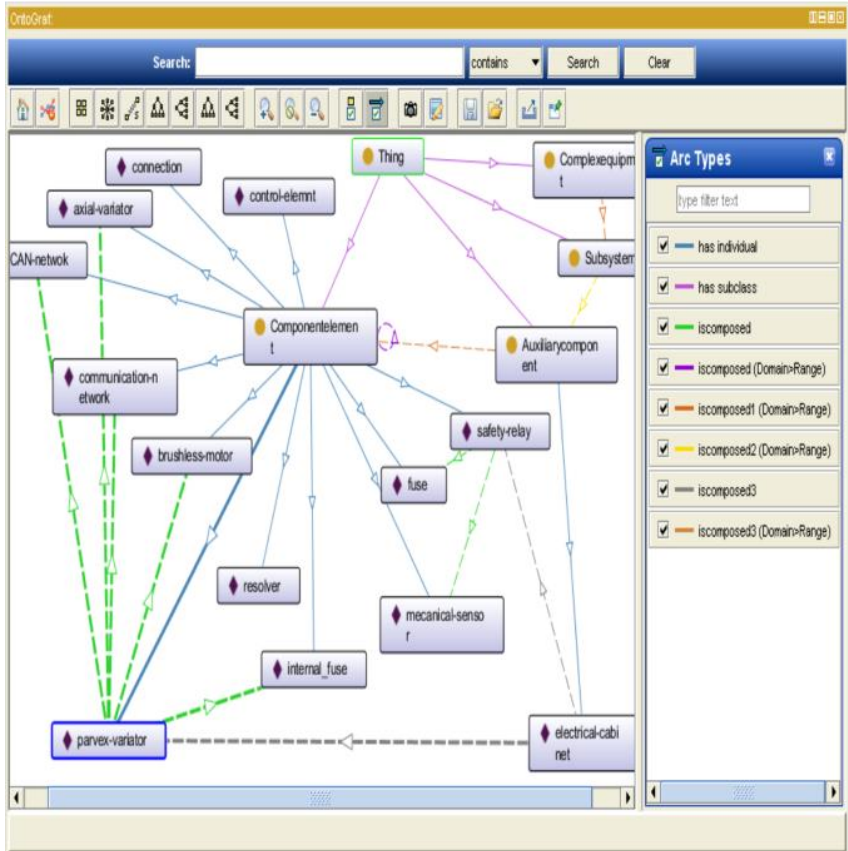

Fig. 7.Partial view of the equipment domain ontology

\section{Operationalization:}

The figure 8 represents the functioning sequence diagram of the proposed organizing module. Ontology 1 is the application domain ontology while ontology 2 represents the equipment domain ontology.

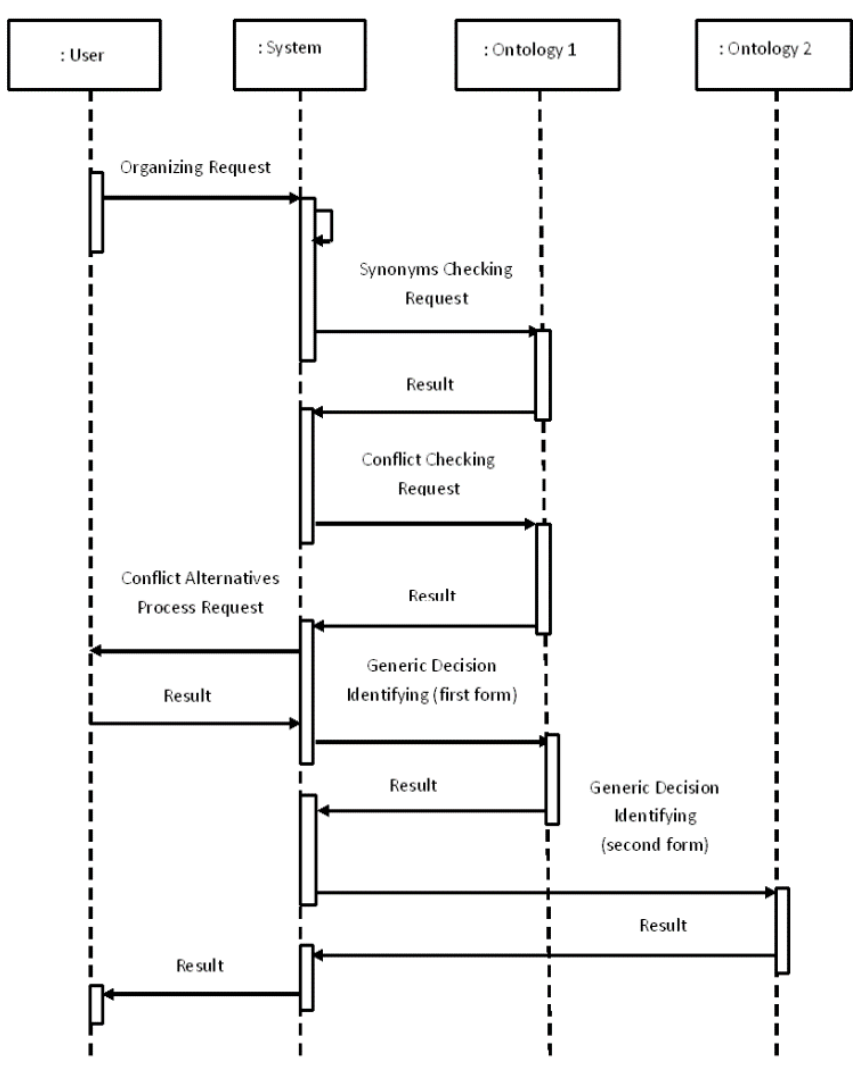


